\title{
Modelo de intención de compra aplicado al sector de Vehículos de Transporte Liviano - Taxis (VTLT)
}

\section{Purchase intention model applied to the Light Transport Vehicle - Taxis (VTLT) sector}

\author{
Martin Malo-Córdova \\ Universidad del Azuay, Cuenca, Ecuador \\ martinmaloc@gmail.com \\ https://orcid.org/0000-0003-1036-2788 \\ Mateo Francisco Ullauri-Ugalde \\ Universidad del Azuay, Cuenca, Ecuador \\ mateou997@gmail.com \\ https://orcid.org/0000-0001-8877-0613 \\ José Leonardo Vera-Reino \\ Universidad del Azuay, Cuenca, Ecuador \\ jvera@uazuay.edu.ec \\ https://orcid.org/0000-0002-4972-7249
}

Recepción: 26/07/2021 | Aceptación: 15/10/2021 | Publicación: 30/10/2021

Cómo citar (APA, séptima edición):

Malo-Córdova, M., Ullauri-Ugalde, M.F., y Vera-R, J. (2021). Modelo de intención de compra aplicado al sector de Vehículos de Transporte Liviano - Taxis (VTLT). INNOVA Research

Journal, 6(3.1), 151-166. https://doi.org/10.33890/innova.v6.n3.1.2021.1856

\section{Resumen}

Entender cuáles son las variables que influyen en la intención de compra de un determinado producto es esencial para proponer estrategias que permitan llegar de mejor manera al cliente. Para esto, el objetivo del presente estudio es proponer un modelo causal que busque medir la intención de compra aplicado al sector de Vehículos de Transporte Liviano - Taxis, y así, determinar algunos de los factores que influyen a la hora de realizar la compra. Para el estudio se aplicaron encuestas a una muestra de 390 taxistas divididos equitativamente en las ciudades de Quito, Guayaquil y Cuenca, cuyo análisis se realizó a través de un análisis factorial confirmatorio y ecuaciones estructurales. Los resultados indicaron que la credibilidad de marca tiene una relación directa e indirecta con todos los factores del estudio, convirtiendo a este factor en uno de los más importantes a tomar en cuenta en el sector de Vehículos de Transporte Liviano - Taxis. Se 
concluye que el consumidor busca confianza y experiencia de la marca a través de la credibilidad en la misma; por lo que los actores de la industria automotriz en el sector deberían enfocar sus recursos para crear una imagen de marcas confiables, haciendo promesas que puedan cumplir.

Palabras claves: credibilidad de marca; intención de compra; marca; vehículo de transporte

liviano.

\begin{abstract}
Understanding which are the variables that influence the purchase intention of a certain product is essential to propose strategies that allow to better reach the customer. For this, the objective of this study is to propose a causal model that seeks to measure the purchase intention applied to the sector of Light Transportation Vehicles - Taxis, and thus, determine some of the factors that influence when making the purchase. For the study, surveys were applied to a sample of 390 taxi drivers divided equally in the cities of Quito, Guayaquil and Cuenca, whose analysis was carried out through a confirmatory factor analysis and structural equations. The results indicated that brand credibility has a direct and indirect relationship with all the factors of the study, making this factor one of the most important to take into account in the Light Transportation Vehicle - Taxis sector. It is concluded that the consumer seeks trust and experience of the brand through its credibility; so the players in the automotive industry in the sector should focus their resources to create an image of trustworthy brands, making promises that they can fulfill.
\end{abstract}

Keywords: brand; brand credibility; purchase intention; transport; vehicles.

\title{
Introducción
}

Entender cuáles son las razones por las que un cliente compra o no un determinado producto es esencial para poder utilizar herramientas de marketing que permitan a las empresas llegar de mejor manera al cliente y saber cuáles son las características que pueden ser mejoradas para ofertar un producto y/o servicio más atractivo. Esta investigación propone un modelo causal que permite analizar algunas de las variables más estudiadas en relación a la intención de compra y determinar la importancia de cada una, basándonos en diversos estudios donde se sustentan algunas de estas relaciones (Baek et al., 2010; DAM, 2020; Hu et al., 2012; Javad, 2016; Khan y Razzaque, 2015).

El sector donde se ha enfocado la investigación es el de los Vehículos de Transporte Liviano - Taxis (VTLT) en el Ecuador, en vista de que son grandes generadores de trabajo. De acuerdo con el diario El Comercio, en promedio entre Quito, Guayaquil y Cuenca hay un taxi por cada 160 habitantes, esto hace que la demanda de vehículos, repuestos y accesorios sea alta, y las empresas vendedoras de vehículos lo saben (El Comercio, 2014). Adicionalmente, estudios dirigidos a este sector en específico, no son comunes y poseen una orientación distinta a nuestro planteamiento. Por lo expuesto, es importante crear un modelo causal de factores que influyen en la intención de compra y probarlo en el sector VTLT, en donde las empresas concesionarias de vehículos podrán conocer algunos factores que el cliente considera y evalúa el momento de decidir realizar una compra, relaciones que analizaremos a continuación.

\section{Marco teórico}

\section{Credibilidad de marca}


La credibilidad de marca se define como credibilidad percibida, es decir la capacidad y la voluntad de cumplir continuamente lo prometido, conformada por dos componentes principales: confiabilidad y experiencia, que pueden llevar a la compra continua de productos por los clientes (Erdem, T. ; Swait, 1998; T. Erdem y Swait, 2004; Gilaninia et al., 2012); por otro lado, el prestigio de marca se define como el posicionamiento relativamente alto de un producto o servicio dentro del mercado al que corresponde; es importante notar que el prestigio de marca no está relacionado con el hecho de que esta sea lujosa (Dubois y Czellar, 2002; Naeyhun Paul Jin et al., 2016; Sataloff et al., 2001). Los estudios de Beak, Kim y Yu , y Paul Jin Naehyun sugieren que la relación entre credibilidad de marca y el prestigio de marca es positiva, es decir, que cuando una marca cumple continuamente con lo prometido está elevando su prestigio; de esta misma manera podemos asumir que si una marca dejara de cumplir con lo prometido, su prestigio dentro del sector se verá afectado de manera negativa (Baek et al., 2010; Hu et al., 2012; Javad Taghipourian et al., 2012; Naehyun (Paul) Jin et al., 2015; Ok et al., 2011; Sataloff et al., 2001). Teniendo en cuenta lo previamente mencionado, se realiza el planteamiento de la primera hipótesis:

\section{H1: La credibilidad de marca tiene relación positiva con el prestigio de marca}

\section{Preferencia de marca}

Preferencia de marca se refiere al agrado del consumidor por el servicio recibido, en comparación con el servicio recibido por otras compañías (Phillip et al., 2003). En la presente investigación no se ha identificado autores que sustenten una relación entre la credibilidad de marca y la preferencia de marca, por lo que se aporta el siguiente planteamiento relacional: marca.

Una relación positiva de credibilidad de marca $\longrightarrow$ calidad percibida $\longrightarrow$ preferencia de

La calidad de marca se define como los juicios del consumidor basándose en los constructos intrínsecos y extrínsecos de los productos o servicios con respecto a la superioridad y excelencia general de las marcas (Aaker, 1991; Bouhlel, 2011; Dubois, 1999; Gilaninia et al., 2012; Sirieix, K.). Sin embargo, la calidad percibida es el juicio del consumidor sobre la superioridad de un producto, es decir, es una percepción abstracta más que un atributo en concreto (Aaker, D.A. y Biel, 2009). Se plantea una relación positiva entre la credibilidad de marca y la calidad percibida debido a que los consumidores emplean una estrategia "Multiple-Cue", es decir, un juicio continuo formado por una serie de variables (Juslin et al., 2003) para juzgar la calidad percibida cuando la credibilidad de marca es alta, lo que quiere decir que la marca puede crear una percepción abstracta de calidad superior (Baek y King 2011; Erdem, T. y Swait 1998; Gotlieb y Sarel 1992). También, la calidad percibida es un aspecto en la equidad de marca (Cobb-Walgren et al., 1995), definida como el valor agregado que un producto tiene por el nombre y lo que representa su marca, dicho esto la relación de calidad percibida con preferencia de marca se sustenta a través de equidad de marca. Esta relación se planteó y probó en el estudio realizado por los autores (Chen y Chang, 2008). Adicionalmente, la relación entre credibilidad de marca y la preferencia de marca puede ser sustentada mediante las siguientes hipótesis, H1 y H5 (explicado posteriormente en el estudio), es decir que, a una mayor credibilidad de marca, mayor prestigio de marca, y un mayor prestigio de marca, mayor preferencia de marca. 
H2: La credibilidad de marca tiene una relación positiva con la preferencia de marca.

\section{Información ahorrada}

Erdem and Swait (1998), afirman que la información ahorrada puede ser conceptualizada por la disminución de información recopilada y costos de procesamiento que incluye dinero, costo psicológico, y gasto del tiempo. También, los consumidores probablemente vean a las marcas creíbles como una fuente de conocimiento para ahorrarse la recopilación de información y los costos de procesamiento, porque les provocará una disminución en la incertidumbre durante el proceso de la decisión de compra (Javad Taghipourian et al., 2012). Por otro lado, se demostró que información ahorrada es uno de varios factores que determinan a una marca como creíble (Baek et al., 2010). Finalmente, el esfuerzo cognitivo necesario para evaluar marcas especificas puede ocasionar una disminución en la probabilidad de selección de la marca (Tülin Erdem y Swait, 2004), por lo que planteamos que la credibilidad de marca disminuye el esfuerzo cognitivo para investigar información por la confianza y experiencia que se tiene con la misma.

H3: La credibilidad de marca tiene una relación positiva con la información ahorrada.

\section{Calidad percibida}

Habiendo explicado el significado de calidad percibida y el significado de prestigio de marca, se sugiere que cuando el prestigio de marca es mayor, la calidad percibida del producto también lo es. Esto ocurre ya que el prestigio de marca es posicionamiento de una marca en un mercado mientras que la calidad percibida son juicios del consumidor sobre un producto o servicio (Dubois y Czellar, 2002; Naeyhun Paul Jin et al., 2016; Sataloff et al., 2001). Por lo tanto, se plantea que cuando un consumidor vea una marca con un prestigio elevado, entonces este consumidor también ve la calidad de esta marca como una calidad elevada (Beneke y Zimmerman, 2014; Javad Taghipourian et al., 2012; Luke y Doke, 1987; Moslehpour y Huyen, 2014). Adicionalmente, en estudios realizados por Baek y Gilaninia en los años 2010 y 2012 respectivamente, se demostró que la relación entre el prestigio de marca y la calidad percibida es positiva (Baek et al., 2010; Gilaninia et al., 2012).

H4: El prestigio de marca tiene una relación positiva con la calidad percibida.

\section{Prestigio de marca}

Roberts y Lattin (1991) sugieren también que la preferencia de marca un proceso que los consumidores se ven expuestos cuando hay una variedad de marcas y una compleja decisión de compra, causando que consumidores eliminan ciertas marcas, las restantes las memorizan para simplificar el proceso y finalmente eligen la más apropiada. La marca seleccionada, en el futuro ahorra este complejo proceso de decisión de compra, siendo más probable su repetida elección (Gensch, 1987; Hwang y Han, 2016; Roberts y Lattin, 1991). Como se menciona en H1, el prestigio de marca se define como el posicionamiento relativamente alto de un producto o servicio dentro del mercado al que corresponde. Consideramos que el prestigio de marca y la preferencia de marca están relacionados positivamente ya que el proceso de selección de una variedad de marcas será más sencillo y rápido, si entre ellas existe una marca con un posicionamiento en el 
mercado superior a las demás. En un estudio realizado en marcas lujosas para viajeros en el año 2015, los autores Hyun y Han plantean una relación positiva entre prestigio de marca y la preferencia de marca; adicionalmente, en el 2016 se realizó otro estudio relacionando las mismas dos variables en la industria de los casinos obteniendo los mismos resultados (Hwang y Han, 2016; Hyun y Han, 2015).

H5: El prestigio de marca tiene una relación positiva con la preferencia de marca.

\section{Intención de compra}

La intención de compra, son actitudes de gusto o disgusto hacia una marca o producto por parte del consumidor y estas deben corresponder completamente a predecir la probabilidad de compra (Torres Valverde, 2013); la intención de compra se aplica como un método para predecir el comportamiento del cliente, analizando la posibilidad de que este realice una compra (Morrison, 1979). Un factor que influye en la intención de compra de los productos es la calidad percibida. La calidad percibida es la valoración que el cliente le entrega a una marca basada en señales intrínsecas y extrínsecas si estas señales son positivas entonces se puede predecir que el comportamiento del cliente tiene una posibilidad de realizar una compra en los productos o servicios de la marca, por lo que la calidad percibida se asocia positivamente con las intenciones de compra (Amiri Aghdaie et al., 2012; Javad, 2016; Javad Taghipourian et al., 2012). En un estudio realizado en el año 2012 se encontró que la calidad percibida es una variable moderadora que se utiliza para saber si los consumidores tienen intención de compra hacia la marca (Gilaninia et al., 2012). El estudio demostró que sí existe dicha relación.

H6: La calidad percibida tiene una relación positiva con la intención de compra.

\section{Preferencia de Marca e Intención de Compra}

Anteriormente, se habló sobre la calidad percibida que es un aspecto fundamental en la equidad de marca y como esta afecta a la preferencia de marca, asimismo Cobb-Walgren (1995) dice que la equidad de marca tiene un fuerte impacto en la intención de compra en los consumidores (Chang y Liu, 2009). Investigaciones realizadas entre 1995 y el 2009, demuestran que los consumidores tienden a comprar productos de su preferencia en varios sectores comerciales, es decir, la preferencia de marca tiene un impacto positivo en la intención de compra (Chang y Liu, 2009; Chen y Chang, 2008; Cobb-Walgren et al., 1995; de Chernatony et al., 2004; Myers, 2003; Prasad y Dev, 2000). Dicha afirmación tiene sentido porque es lógico suponer que un cliente tendrá mayor intención de compra hacia una marca de su preferencia ya sea por experiencia con la marca, resultados de la marca, credibilidad de la marca y/o el prestigio de ésta. Por ejemplo, en un estudio realizado en el año 2011 se plantea la relación positiva entre preferencia de marca e intención de compra donde el país de procedencia actúa como moderador entre estas dos variables. El estudio evidenció que sí existe una relación entre ambas variables (Moradi y Zarei, 2011).

H7: La preferencia de marca tiene un impacto positivo en la intención de compra.

\section{Información Ahorrada e Intención de Compra}

Esta obra se comparte bajo la licencia Creative Common Atribución-No Comercial 4.0 International (CC BY-NC 4.0) 
La intención de compra también es afectada de manera positiva por la información ahorrada, ya que la información ahorrada implica que existe un menor riesgo para el consumidor al realizar una compra. Es decir, que el consumidor tiene menos incertidumbre sobre el producto o servicio ofrecido por la marca cuando tiene un alto conocimiento de la misma, lo que eleva las posibilidades de que el consumidor efectivamente realice la compra del producto o servicio ofrecido por la marca (Baek et al., 2010; Gilaninia et al., 2012; Javad Taghipourian et al., 2012). Un estudio realizado en el año 2011 donde se midieron los efectos de la credibilidad de marca en distintas categorías de servicio sustenta la hipótesis, concluyendo que la información ahorrada afecta positivamente a la intención de compra siempre y cuando el consumidor tenga una credibilidad de marca fuerte (Baek y King, 2011). Adicionalmente, en el 2015 los autores M. Khan y R. Razzaque demostraron que cuando la información ahorrada es alta, los consumidores se inclinan más a comprar ese producto ya que la información ahorrada conduce a una deducción de la calidad del producto ofrecido por la marca (Khan y Razzaque, 2015).

H8: La información ahorrada tiene un impacto positivo en la intención de compra.

Luego de haber expuesto el fundamento teórico sobre el cual se han planteado las hipótesis, la Figura 1 muestra el modelo causal propuesto.

\section{Figura 1}

\section{Modelo causal}

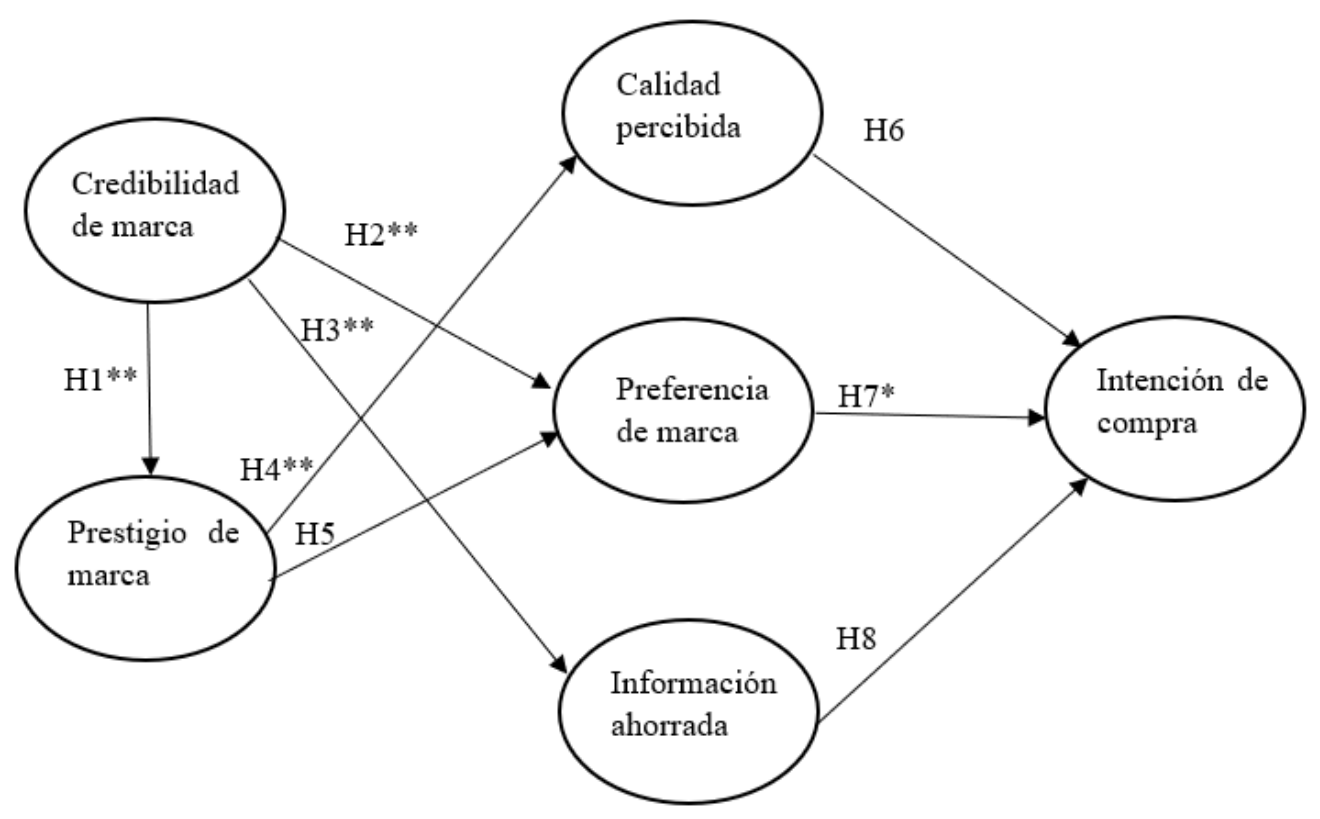

\section{Metodología}

Para plantear nuestro modelo causal, se realizó una búsqueda de otros modelos válidos y fiables que utilicen algunas de las principales variables que afecten la intención de compra. Adicionalmente, se realizaron entrevistas a ejecutivos de nivel superior del sector automotriz 
ecuatoriano, para tener una idea de cuanto conocimiento hay sobre este tema en el sector. En dichas entrevistas se logró identificar que existe un desconocimiento generalizado en el sector VTLT, donde la gran mayoría de las decisiones y estrategias se realizan de forma empírica. Utilizando estas referencias se procedió a crear un modelo causal con las variables que se combinaron de los modelos propuestos por los autores Hu, Liu, Wang \& Yang (2012) de donde se obtuvo la variable "Preferencia de Marca" y Baek, Kim y Yu (2010) de donde se obtuvo las variables; "Credibilidad de marca", "Calidad percibida", "Información ahorrada", "Prestigio de marca" e "Intención de compra"

\section{Tabla 1}

Detalle de las escalas utilizadas en la investigación

\section{Variables Indicadores Pregunta}

\begin{tabular}{|c|c|c|}
\hline \multirow{3}{*}{$\begin{array}{l}\text { Prestigio de } \\
\text { marca }\end{array}$} & BPR1 & Es de una marca prestigiosa \\
\hline & BPR2 & Es de una marca que tiene un alto estatus \\
\hline & BPR3 & Es de una marca muy exclusiva \\
\hline \multirow{2}{*}{$\begin{array}{l}\text { Calidad } \\
\text { percibida }\end{array}$} & PQ1 & Tiene la mejor calidad \\
\hline & PQ2 & Le doy una buena calificación \\
\hline \multirow{3}{*}{$\begin{array}{l}\text { Preferencia } \\
\text { de marca }\end{array}$} & $\mathrm{BP} 2$ & Se adapta a mis necesidades \\
\hline & BP3 & Es atractiva para mis compañeros \\
\hline & $\mathrm{BC} 1$ & Entrega lo que promete \\
\hline \multirow{4}{*}{$\begin{array}{l}\text { Credibilidad } \\
\text { de marca }\end{array}$} & BC3 & Con el tiempo entendí que si cumple con lo que promete \\
\hline & $\mathrm{BC} 4$ & Se esfuerza por entregarte lo que te ofrecieron \\
\hline & BC5 & Puedes confiar en el nombre de la marca \\
\hline & BC6 & Se que puedo confiar en la marca en el futuro \\
\hline \multirow{3}{*}{$\begin{array}{l}\text { Información } \\
\text { ahorrada }\end{array}$} & ICS1 & Saber lo que la marca ofrece, me ahorra tiempo al comprar \\
\hline & ICS2 & Necesito mucha información de la marca antes de comprarla \\
\hline & ICS3 & Para saber que tal es esta marca, tendía que usarla varias vece \\
\hline \multirow{3}{*}{$\begin{array}{l}\text { Intención de } \\
\text { compra }\end{array}$} & PI1R & Nunca compraría esta marca \\
\hline & PI2 & Seriamente consideraría comprar la marca \\
\hline & PI3 & ¿Qué tan probable fuera que compres la marca de tu taxi? \\
\hline
\end{tabular}

Nota: El cuestionario contó con estas preguntas utilizando una escala de Likert de siete puntos.

El modelo causal propuesto se testeó a través de 390 encuestas aplicadas equitativamente a taxistas en las ciudades de Quito, Guayaquil y Cuenca.

Los datos obtenidos fueron analizados en SPSS para obtener la fiabilidad a través del coeficiente de alpha de Cronbach. Luego se procedió a utilizar EQS para el análisis factorial confirmatorio y ecuaciones estructurales para el testeo de las hipótesis.

\section{Resultados y discusión}

\section{Resultados}


En el análisis de los datos se utilizó la metodología recomendada por Anderson y Gerbing (1988) en la cual primero se realizó un análisis factorial confirmatorio (AFC), y luego un análisis estructural del modelo. Se escogió esta metodología para primero probar la validez y fiabilidad del estudio, así como su ajuste, para posteriormente analizar las relaciones estructurales del modelo y comprobar las hipótesis (Anderson y Gerbing, 1988).

Observamos que las cargas estandarizadas eran bajas, por lo que se retiraron algunas variables y se procedió a replantear el modelo. Esto se debe a que las variables utilizadas son socialmente sensibles y no se adaptaron de manera correcta. Con esto, se obtuvieron los resultados que se utilizaron para el modelo final, como se observa en la Tabla 2.

En el estudio, la prueba del Chi-cuadrado (Tabla 2) es utilizada para probar el efecto de ajuste absoluto, identificando los grados de libertad del modelo teórico y del modelo observado, y explicando la importancia demostrativa del modelo. Para tener un buen ajuste se requiere de un chi cuadrado no significativo, y en la matriz residual de covarianzas se necesita que los residuos se agrupen hacia el centro del grafico (Bagozzi y Yi, 2012; Civelek, 2018; Wu et al., 2018).

Como se puede observar en la Tabla 2, se obtuvo el índice RMSEA (Root Mean Square of Error Aproximation) que provee información sobre el ajuste, se recomienda que este valor sea menor a 0.08 , en nuestro caso, con un valor de 0.074 el ajuste se encuentra dentro de parámetros aceptables (Taasoobshirazi y Wang, 2016). Aplicamos el índice CFI (Comparative Fix Index) que al igual del RMSEA mide el ajuste del modelo con la diferencia de que el valor debe ser superior a 0.9 para ser aceptado. Nuestro modelo tuvo un CFI de 0.93 (Jones et al., 2010; Taasoobshirazi y Wang, 2016).

En los resultados, no hubo correlaciones superiores a la unidad ni cargas factoriales estandarizadas fuera del intervalo $(-1,+1)$. No se encontraron estimaciones negativas en las varianzas y las estimaciones son significativas.

Para evaluar la fiabilidad de los datos se realizó un análisis de coeficiente del alfa de Cronbach (CA) y la fiabilidad compuesta del constructo (CR), para ambos índices se aceptan resultados sobre el 0.7 de acuerdo con la metodología (Nunnally, 1978; Ramírez et al., 2014). Para evaluar la validez del estudio, se utilizó la Varianza Extraída Media (AVE) que, según Fornell y Lacker (1981) debe ser igual o superior a 0.5 para ser aceptable en un AFC (Chin, 1998; Fornell y Larcker, 1981).

Tabla 2

Variables que influyen en la intención de compra

\begin{tabular}{|c|c|c|c|c|c|c|c|}
\hline Variables & & Indicadores & $\begin{array}{c}\text { Cargas } \\
\text { estandarizadas }\end{array}$ & Valor $\mathbf{t}$ & $\mathbf{C A}$ & CR & AVE \\
\hline \multirow{4}{*}{$\begin{array}{l}\text { Prestigio } \\
\text { marca }\end{array}$} & \multirow{4}{*}{ de } & BPR1 & $0.637 * *$ & 13.410 & 0.74 & 0.75 & 0.50 \\
\hline & & BPR2 & $0.703 * *$ & 15.199 & & & \\
\hline & & BPR3 & $0.773 * *$ & 17.220 & & & \\
\hline & & PQ1 & $0.812 * *$ & 18.690 & 0.84 & 0.84 & 0.72 \\
\hline
\end{tabular}




\begin{tabular}{|c|c|c|c|c|c|c|}
\hline Variables & Indicadores & $\begin{array}{c}\text { Cargas } \\
\text { estandarizadas }\end{array}$ & Valor $\mathbf{t}$ & CA & CR & AVE \\
\hline $\begin{array}{l}\text { Calidad } \\
\text { percibida }\end{array}$ & PQ2 & $0.888 * *$ & 21.210 & & & \\
\hline \multirow{2}{*}{$\begin{array}{l}\text { Preferencia de } \\
\text { marca }\end{array}$} & BP2 & $0.746 * *$ & 15.255 & \multirow{2}{*}{0.69} & \multirow{2}{*}{0.74} & \multirow[t]{2}{*}{0.59} \\
\hline & BP3 & $0.789 * *$ & 16.206 & & & \\
\hline \multirow{5}{*}{$\begin{array}{l}\text { Credibilidad de } \\
\text { marca }\end{array}$} & $\mathrm{BC} 1$ & $0.788 * *$ & 18.000 & \multirow[t]{5}{*}{0.87} & \multirow[t]{5}{*}{0.87} & \multirow[t]{5}{*}{0.58} \\
\hline & $\mathrm{BC} 3$ & $0.687 * *$ & 14.858 & & & \\
\hline & $\mathrm{BC} 4$ & $0.707 * *$ & 15.464 & & & \\
\hline & BC5 & $0.786^{* *}$ & 17.928 & & & \\
\hline & BC6 & $0.823 * *$ & 19.229 & & & \\
\hline \multirow{3}{*}{$\begin{array}{l}\text { Información } \\
\text { ahorrada }\end{array}$} & ICS1 & $0.710 * *$ & 14.625 & \multirow[t]{3}{*}{0.79} & \multirow[t]{3}{*}{0.79} & \multirow[t]{3}{*}{0.56} \\
\hline & ICS2 & $0.786 * *$ & 16.608 & & & \\
\hline & ICS3 & $0.739 * *$ & 15.390 & & & \\
\hline \multirow{3}{*}{$\begin{array}{l}\text { Intención } \\
\text { compra }\end{array}$} & PI1R & $0.747 * *$ & 16.089 & \multirow[t]{3}{*}{0.85} & \multirow[t]{3}{*}{0.85} & \multirow[t]{3}{*}{0.65} \\
\hline & PI2 & $0.850 * *$ & 18.927 & & & \\
\hline & PI3 & $0.819 * *$ & 18.036 & & & \\
\hline \multicolumn{7}{|c|}{$\begin{array}{l}\mathrm{N}=390 ; \mathrm{X}^{2}(120 \mathrm{df})=377.39(p>.05) ; \mathrm{NFI}=0.90 ; \mathrm{NNFI}=0.91 ; \mathrm{CFI}=0.93 ; \mathrm{IFI}=0.93 ; \\
\text { RMSEA }=0.074 \\
\text { Notas: } * p<.05 ; * * p<.01 ; \mathrm{CA}=\alpha \text { de Cronbach; } \mathrm{CR}=\text { fiabilidad compuesta; } \mathrm{AVE}=\text { varianza } \\
\text { extraida promedio. }\end{array}$} \\
\hline
\end{tabular}

Se determinó que el modelo tiene validez convergente ya que las cargas de los factores son significativas una vez que el modelo fue rectificado como se mencionó anteriormente (Bagozzi y Yi, 1988). Parte del estudio involucró determinar si el instrumento de medida tiene una validez discriminante para lo que se utilizaron dos metodologías: el test de intervalos de confianza y el test de varianza extraída (Anderson y Gerbing, 1988; Fornell y Larcker, 1981). En ambos casos, los resultados indican que el instrumento de medida en términos generales tiene validez discriminante (ver Tabla 3).

\section{Tabla 3}

Validez discriminante e intervalos de valor

\begin{tabular}{|c|c|c|c|c|c|c|}
\hline & 1 & 2 & 3 & 4 & 5 & 6 \\
\hline Prestigio de marca & 0.50 & 0.97 & 0.40 & 0.51 & 0.26 & 0.10 \\
\hline Calidad percibida & $(0.94 ; 1.03)$ & 0.72 & 0.32 & 0.43 & 0.18 & 0.05 \\
\hline $\begin{array}{l}\text { Preferencia de } \\
\text { marca }\end{array}$ & $(0.53 ; 0.73)$ & $(0.47 ; 0.66)$ & 0.59 & 0.62 & 0.28 & 0.09 \\
\hline $\begin{array}{l}\text { Credibilidad } \\
\text { marca }\end{array}$ & $(0.64 ; 0.79)$ & $(0.58 ; 0.73)$ & $(0.72 ; 0.85)$ & 0.58 & 0.48 & 0.06 \\
\hline $\begin{array}{l}\text { Informacion } \\
\text { ahorrada }\end{array}$ & $(0.41 ; 0.62)$ & $(0.32 ; 0.53)$ & $(0.42 ; 0.63)$ & $(0.62 ; 0.77)$ & 0.56 & 0.06 \\
\hline Intencion de compra & $(0.20 ; 0.43)$ & $(0.11 ; 0.34)$ & $(0.18 ; 0.41)$ & $(0.15 ; 0.37)$ & $(0.13 ; 0.36)$ & 0.65 \\
\hline
\end{tabular}

Notas: En la diagonal encontramos las varianzas extraídas, en el triángulo inferior el intervalo de confianza entre los indicadores, y en el triángulo superior encontramos las correlaciones al cuadrado. 
Luego se procedió a testear las hipótesis del modelo estructural propuesto, en este análisis se determinó que la credibilidad de marca influye de manera positiva en el prestigio de marca (H1) $(\beta=0.750, \mathrm{p}<0.01)$, así también se determinó que la credibilidad de marca influye de manera positiva en la preferencia de marca $(\mathrm{H} 2)(\beta=0.743, \mathrm{p}<0.01)$, la credibilidad de marca influye de manera positiva en la información ahorrada $(\mathrm{H} 3)(\beta=0.758, \mathrm{p}<0.01)$, el prestigio de marca influye de manera positiva a la calidad percibida (H4) $(\beta=0.976, p<0.01)$, y que la preferencia de marca influye de manera positiva en la intención de compra $(\mathrm{H} 7)(\beta=0.750, \mathrm{p}<0.05)$. Los resultados se muestran en la Tabla 4.

Se puede observar en la Figura 1 que la credibilidad de marca influye de manera indirecta en la calidad percibida a través del prestigio de marca $(\mathrm{H} 1$ y H4), así como en la intención de compra a través de la preferencia de marca $(\mathrm{H} 2$ y $\mathrm{H} 7)$.

\section{Tabla 4}

Testeo de Hipótesis

\begin{tabular}{|c|c|c|c|}
\hline Hipótesis & & $\beta$ estandarizado & Valor $\mathbf{t}$ \\
\hline H1 & Credibilidad $\longrightarrow$ Prestigio de marca & $0.750 * *$ & 12.321 \\
\hline H2 & Credibilidad $\longrightarrow$ Preferencia de marca & $0.743 * *$ & 9.745 \\
\hline H3 & Credibilidad $\longrightarrow$ Información ahorrada & $0.758 * *$ & 13.194 \\
\hline H4 & Prestigio de marca $\longrightarrow$ Calidad percibida & $0.976 * *$ & 13.749 \\
\hline H5 & Prestigio de marca $\longrightarrow$ Preferencia de marca & 0.121 & 1.706 \\
\hline H6 & Calidad percibida $\longrightarrow$ Intención de compra & 0.112 & 1.351 \\
\hline H7 & Preferencia de marca $\longrightarrow$ Intención de compra & $0.201 *$ & 2.025 \\
\hline H8 & Información ahorrada $\rightarrow$ Intención de compra & 0.106 & 1.258 \\
\hline \multicolumn{4}{|c|}{$\begin{array}{l}X^{2}(128 \mathrm{df})=424.131 ;(p<.05) ; \text { RMSEA }(90 \% \mathrm{CI})=0.077(0.069,0.085) ; \mathrm{CFI}=.919 ; \\
\mathrm{NNFI}=.903 \\
* * p<.01 ; * p<.05\end{array}$} \\
\hline
\end{tabular}

\section{Discusión}

La relación entre credibilidad y prestigio de marca $(\mathrm{H} 1)$ se aceptó en nuestro estudio como también ha sido aceptada en estudios anteriores (Baek et al., 2010; Baek y King, 2011; N. Jin et al., 2015), en donde se confirma que la credibilidad de marca influye positivamente en el prestigio de marca, es decir, cuando un cliente cree en lo que una marca ofrece, el posicionamiento de la misma será más elevado en el mercado y cuando una marca no cumple con lo prometido su prestigio se verá afectado.

En el caso de la credibilidad y la preferencia de marca $(\mathrm{H} 2)$ se acepta la relación, esto era de esperar porque cuando una marca está constantemente cumpliendo con lo prometido genera un favoritismo por parte del consumidor hacia la misma (Pecot y De Barnier, 2018), además, Philip (2003) menciona a la preferencia de marca como una comparación que los consumidores realizan 
del servicio recibido de varias marcas, en donde es natural suponer que preferir una marca es consecuencia de sus dos componentes principales: confiabilidad y experiencia que se ha tenido con la misma (Erdem, T. ; Swait, 1998; Tülin Erdem y Swait, 2004; Gilaninia et al., 2012; Phillip et al., 2003).

En cuanto a la información ahorrada y su relación con la credibilidad de marca (H3) no nos sorprende que la hipótesis se acepte, ya que varios autores han demostrado que existe dicha relación (Baek et al., 2010; Baek y King, 2011; Tülin Erdem y Swait, 2004; Hwang y Hyun, 2012; Javad Taghipourian et al., 2012; N. Jin et al., 2015; Xu, 2019). Esto es lógico porque el creer en una marca disminuye el proceso de recopilación de información y los costos de procesamiento, como costos psicológicos y gastos de tiempo por parte de los consumidores. También, la credibilidad que genera una marca disminuye el esfuerzo cognitivo por la experiencia que se tiene de ella.

La relación entre prestigio y calidad de marca $(\mathrm{H} 4)$ se sustenta por medio de varios estudios (Baek et al., 2010; Gilaninia et al., 2012; Javad Taghipourian et al., 2012; Ji et al., 2018), esto tiene sentido, ya que la calidad percibida de la marca está basada en los constructos intrínsecos y extrínsecos con respecto a la superioridad y excelencia general de la marca, ese decir, el prestigio de la marca (Aaker, 1991; Bouhlel, 2011). Por otro lado, la calidad percibida es una percepción abstracta del cliente causado por el prestigio de una marca dentro del mercado.

Si bien se plantea la relación de prestigio y preferencia de marca (H5), esta no se acepta pese a que otros autores han demostrado que existe una relación fuerte entre estas dos variables (Casidy et al., 2018; Hwang y Han, 2016; Hyun y Han, 2015). Esto puede estar ligado a factores económicos de los consumidores a lo que se los orientó el estudio. Los autores que afirman la relación hicieron sus estudios con marcas lujosas para viajeros (2015) y con casinos (2016) que son industrias que captan consumidores económicamente fuertes, mientras que nuestro estudio se enfocó en consumidores y mercados económicamente más restringidos. Además, podemos suponer que los encuestados no prefieren una marca prestigiosa, sino que son influenciados por otros factores como precio.

Pese a que varios autores sustentan una relación positiva entre la calidad percibida y la intención de compra (H6), en nuestro estudio esta no se acepta (Amiri Aghdaie et al., 2012; Gilaninia et al., 2012; Javad, 2016; Javad Taghipourian et al., 2012; J. Wang et al., 2020). Estudios han demostrado que el comportamiento del cliente hacia una marca depende del tipo de economía a la que pertenece (desarrollada o en desarrollo), por un lado, en países con economías fuertes el cliente presta más atención a la calidad, mientras que en países con economías en desarrollo el precio es un factor determinante. Suponemos que la hipótesis no se cumple por factores socioeconómicos de los mercados y consumidores donde fueron realizadas las encuestas (Bilan et al., 2019).

La hipótesis que plantea una relación entre preferencia de marca e intención de compra (H7) fue aceptada, algo esperado ya que varias investigaciones han demostrado una tendencia de los consumidores a comprar productos de marcas de su preferencia (Boubker y Douayri, 2020; Chang y Liu, 2009; Chen y Chang, 2008; Cobb-Walgren et al., 1995; de Chernatony et al., 2004; Moradi y Zarei, 2011; Myers, 2003; Prasad y Dev, 2000), por lo que las marcas que invierten en 
campañas de mejora percepción y preferencia en el mercado, observan un incremento en la intención de compra de posibles consumidores (DAM, 2020). El preferir una marca, que como se sustentó anteriormente, obedece en parte a su credibilidad, es un proceso complejo dado por factores ligados a la confiabilidad, experiencia y percepción de la marca en el mercado.

Para la hipótesis H8 varios autores han demostrado la relación entre información ahorrada e intención de compra (Baek y King, 2011; Khan y Razzaque, 2015; H. J. Wang, 2017), sin embargo, en nuestro estudio no se aceptó dicha relación. La información ahorrada significa que el cliente conoce la marca, sin embargo, esto no quiere decir que el consumidor esté dispuesto a comprar dicha marca, ya que su conocimiento puede plantear una imagen negativa de los productos o servicios ofrecidos, o pueden existir barreras de acceso como precio o regulaciones locales, en conclusión, conocer una marca no es indicativo de comprarla.

\section{Conclusiones}

En esta investigación se buscó comprender cuáles son las razones que influyen en la intención de compra en el sector VTLT. Para este fin se plantearon tres hipótesis alrededor de la intención de compra, en donde se aceptó la relación con preferencia de marca, y se rechazaron las relaciones con calidad percibida y con información ahorrada. El rechazo de la relación entre calidad percibida e intención de compra puede deberse a que la calidad percibida no es un factor determinante en la intención de compra en una economía en vías de desarrollo (Bilan et al., 2019). Mientras que el rechazo de la relación entre información ahorrada e intención de compra puede obedecer a que mucho conocimiento sobre la marca no es un factor determinante de su intención de compra en esta categoría de producto.

En las relaciones complementarias de la investigación se aceptó la relación entre credibilidad de marca y prestigio de marca; credibilidad de marca con preferencia de marca; credibilidad de marca con información ahorrada; y prestigio de marca con calidad percibida. Por otro lado, no se aceptó la relación entre prestigio de marca y preferencia. Esto puede estar ligado a factores económicos de los consumidores a los que se orientó el estudio. Además, podemos suponer que los encuestados no prefieren una marca prestigiosa, sino que son influenciados por otros factores, por lo que, en futuras investigaciones consideraríamos añadir el factor precio como moderador del modelo. También se recomienda otra investigación utilizando este modelo para que se tengan datos con que comparar.

Adicionalmente, la credibilidad de marca tiene una relación directa e indirecta con todos los factores del estudio, convirtiendo a este factor en la base fundamental del sector VTLT. Basándonos en esto, podemos concluir que el consumidor del sector VTLT busca confianza y experiencia de la marca en el sector, al ser estas dos variables componentes de la credibilidad. Finalmente, sugerimos que las marcas del sector automotriz en el sector VTLT estructuren planes, que logren captar la atención de los consumidores, como marcas especializadas en el sector VTLT.

\section{Referencias bibliográficas}

Aaker, D.A.; Biel, A. . (2009). Brand equity \& Advertising (A. . Aaker, D.A.; Biel (ed.)). Lawrence Erlbaum Associates, Inc. 
Aaker, D. A. (1991). Managing Brand Value: Capitalizing on the Value of a Brand Name. The Free Press, New York.

Amiri Aghdaie, S. F., Dolatabadi, H. R., \& Aliabadi, V. S. (2012). An Analysis of Impact of Brand Credibility and Perceived Quality on Consumers' Evaluations of Brand Alliance. International Journal of Marketing Studies, 4(2), 93-102. https://doi.org/10.5539/ijms.v4n2p93

Anderson, J. C., \& Gerbing, D. W. (1988). Structural Equation Modeling in Practice : A Review and Recommended Two-Step Approach. 103(3), 411-423.

Baek, T. H., Kim, J., \& Yu, J. H. (2010). The differential roles of brand credibility and brand prestige in consumer brand choice. Psychology and Marketing, 27(7), 662-678. https://doi.org/10.1002/mar.20350

Baek, T. H., \& King, K. W. (2011). Exploring the consequences of brand credibility in services. Journal of Services Marketing, 25(4), 260-272. https://doi.org/10.1108/08876041111143096

Bagozzi, R. P., \& Yi, Y. (1988). On the evaluation of structural equation models. Journal of the Academy of Marketing Science, 16(1), 74-94. https://doi.org/10.1007/BF02723327

Bagozzi, R. P., \& Yi, Y. (2012). Specification, evaluation, and interpretation of structural equation models. Journal of the Academy of Marketing Science, 40(1), 8-34. https://doi.org/10.1007/s11747-011-0278-x

Beneke, J., \& Zimmerman, N. (2014). Beyond private label panache: The effect of store image and perceived price on brand prestige. Journal of Consumer Marketing, 31(4), 301-311. https://doi.org/10.1108/JCM-12-2013-0801

Bilan, Y., Lyeonov, S., Lyulyov, O., \& Pimonenko, T. (2019). Brand management and macroeconomic stability of the country. Polish Journal of Management Studies, 19(2), 6174. https://doi.org/10.17512/pjms.2019.19.2.05

Boubker, O., \& Douayri, K. (2020). Dataset on the relationship between consumer satisfaction, brand attitude, brand preference and purchase intentions of dairy product: The case of the Laayoune-Sakia El Hamra region in Morocco. Data in Brief, 32, 0-6. https://doi.org/10.1016/j.dib.2020.106172

Bouhlel, O. (2011). Brand Personality's Influence on the Purchase Intention: A Mobile Marketing Case. 6(9), 210-228. https://doi.org/10.5539/ijbm.v6n9p210

Casidy, R., Nyadzayo, M., Mohan, M., \& Brown, B. (2018). The relative influence of functional versus imagery beliefs on brand sensitivity in B2B professional services. Industrial Marketing Management, $\quad$ 72(July 2017), 26-36. https://doi.org/10.1016/j.indmarman.2018.01.021

Chang, H. H., \& Liu, Y. M. (2009). The impact of brand equity on brand preference and purchase intentions in the service industries. Service Industries Journal, 29(12), 1687-1706. https://doi.org/10.1080/02642060902793557

Chen, C. F., \& Chang, Y. Y. (2008). Airline brand equity, brand preference, and purchase intentions-The moderating effects of switching costs. Journal of Air Transport Management, 14(1), 40-42. https://doi.org/10.1016/j.jairtraman.2007.11.003

Chin, W. W. (1998). The partial least squares approach to structural equation modelling. In Marcoulides G. A. (Ed.). Modern Methods for Business Research, 295(2), 295-336.

Civelek, M. E. (2018). Essentials of Structural Equation Modeling. In Zea Books. https://doi.org/10.13014/k2sj1hr5

Cobb-Walgren, C. J., Ruble, C. A., \& Donthu, N. (1995). Brand equity, brand preference, and purchase intent. Journal of Advertising, 24(3), 25-40. 
https://doi.org/10.1080/00913367.1995.10673481

DAM, T. C. (2020). Influence of Brand Trust, Perceived Value on Brand Preference and Purchase Intention. Journal of Asian Finance, Economics and Business, 7(10), 939-947. https://doi.org/10.13106/jafeb.2020.vol7.no10.939

de Chernatony, L., Harris, F. J., \& Christodoulides, G. (2004). Developing a brand performance measure for financial services brands. Service Industries Journal, 24(2), 15-33. https://doi.org/10.1080/02642060412331301232

Dubois, B., \& Czellar, S. (2002). Prestige Brands or Luxury Brands? An Exploratory Inquire on Consumer Perceptions. European Marketing Academy, 1-9.

El Comercio. (2014). En el país hay sobreoferta de taxis, pero el negocio aún resulta rentable. El Comercio.

Erdem, T.; Swait, J. (1998). Brand equity as a signaling phenomenon. Journal of Consumer Psychology, 7(2), 131-157.

Erdem, T., \& Swait, J. (2004). Brand credibility, brand consideration, and choice. Journal of Consumer Research, 191-198. https://doi.org/10.1086/383434

Erdem, Tülin, \& Swait, J. (2004). Brand Credibility, Brand Consideration, and Choice. 31(June).

Fornell, C., \& Larcker, D. F. (1981). SEM with Unobservable Variables and Measurement Error: Algebra and Statistics. In Journal of Marketing Research 18(3), 1-16. https://doi.org/10.2307/3150980

Gensch, D. H. (1987). A Two-Stage Disaggregate Attribute Choice Model. Marketing Science, 6(3), 223-239. https://doi.org/10.1287/mksc.6.3.223

Gilaninia, S., Ganjinia, H., Moridi, A., \& Rahimi, M. (2012). The differential roles of brand credibility and brand prestige in the customers' purchase intention. Kuwait Chapter of Arabian Journal of Business and Management Review. 2(4), 1-10. https://www.arabianjbmr.com/pdfs/KD_VOL_2_4/1.pdf

Gotlieb, J. B., \& Sarel, D. (1992). The Influence of Type of Advertisement, Price, and Source Credibility on Perceived Quality. Journal of the Academy of Marketing Science, 20(3), 253260. https://doi.org/10.1177/0092070392203006

Hu, J., Liu, X., Wang, S., \& Yang, Z. (2012). The role of brand image congruity in Chinese consumers' brand preference. Journal of Product and Brand Management, 21(1), 26-34. https://doi.org/10.1108/10610421211203088

Hwang, J., \& Han, H. (2016). A Model of Brand Prestige Formation in the Casino Industry. Journal of Travel and Tourism Marketing, 33(8), 1106-1123. https://doi.org/10.1080/10548408.2015.1084977

Hwang, J., \& Hyun, S. S. (2012). The Antecedents and Consequences of Brand Prestige in Luxury Restaurants. Asia Pacific Journal of Tourism Research, 17(6), 656-683. https://doi.org/10.1080/10941665.2011.640697

Hyun, S. S., \& Han, H. (2015). Luxury Cruise Travelers: Other Customer Perceptions. https://doi.org/10.1177/0047287513513165

Javad, K. (2016). The Effects of Brand Credibility and Prestige on Consumers Purchase Intention in Low and High Product Involvement The Effects of Brand Credibility and Prestige on Consumers Purchase. January 2012.

Javad Taghipourian, M., Author, C., \& Heidarzadeh Hanzaee, K. (2012). The Effects of Brand Credibility and Prestige on Consumers Purchase Intention in Low and High Product Involvement. J. Basic. Appl. Sci. Res, 2(2), 1281-1291. https://www.researchgate.net/publication/301613897 
Ji, C., Zeng, Z., \& Kale, S. H. (2018). The antecedents of tourists' gaming spend: does the brand prestige matter? Asia Pacific Journal of Tourism Research, 23(11), 1086-1097. https://doi.org/10.1080/10941665.2018.1524775

Jin, N., Lee, S., \& Jun, J. H. (2015). The role of brand credibility in predicting consumers' behavioural intentions in luxury restaurants. Anatolia, 26(3), 384-396. https://doi.org/10.1080/13032917.2014.969284

Jin, Naehyun (Paul), Lee, S. (Shawn), \& Jun, J. H. (2015). The role of brand credibility in predicting consumers' behavioural intentions in luxury restaurants. Anatolia, 26(3), 384-396. https://doi.org/10.1080/13032917.2014.969284

Jin, Naeyhun (Paul), Line, N. D., \& Merkebu, J. (2016). The Impact of Brand Prestige on Trust, Perceived Risk, Satisfaction, and Loyalty in Upscale Restaurants. Journal of Hospitality Marketing and Management, 25(5), 523-546. https://doi.org/10.1080/19368623.2015.1063469

Jones, A. E., Shapiro, N. I., Trzeciak, S., Arnold, R. C., Claremont, H. A., \& Kline, J. A. (2010). Lactate clearance vs central venous oxygen saturation as goals of early sepsis therapy: A randomized clinical trial. JAMA - Journal of the American Medical Association, 303(8), 739746. https://doi.org/10.1001/jama.2010.158

Juslin, P., Olsson, H., \& Olsson, A. C. (2003). Exemplar Effects in Categorization and MultipleCue Judgment. Journal of Experimental Psychology: General, 132(1), 133-156. https://doi.org/10.1037/0096-3445.132.1.133

Khan, M. M., \& Razzaque, R. (2015). Measuring the impact of brand positioning on consumer purchase intention across. XI(I), 69-95.

Luke, R. H., \& Doke, E. R. (1987). Marketing journal hierarchies: Faculty perceptions, 1986-87. Journal of the Academy of Marketing Science, 15(1), 74-78. https://doi.org/10.1007/BF02721957

Moradi, H., \& Zarei, A. (2011). The impact of brand equity on purchase intention and brand preference-the moderating effects of country of origin image. Australian Journal of Basic and Applied Sciences, 5(3), 539-545.

Morrison, D. G. (1979). Purchase intentions and purchase behavior. Journal of Marketing 43(2), 65-74. https://doi.org/10.2307/1250742

Moslehpour, M., \& Huyen, N. T. Le. (2014). The Influence of Perceived Brand Quality and Perceived Brand Prestige on Purchase Likelihood of iPhone and HTC Mobile Phone in Taiwan. Research in Business and Management, 1(1), 62. https://doi.org/10.5296/rbm.v1i1.4882

Myers, C. A. (2003). Managing brand equity: A look at the impact of attributes. Journal of Product \& Brand Management, 12(1), 39-51. https://doi.org/10.1108/10610420310463126

Nunnally, J. (1978). An Overview of Psychological Measurement. Agile Project Management with Azure DevOps, 37-66. https://doi.org/10.1007/978-1-4842-4483-8_2

Ok, C., Choi, Y. G., \& Hyun, S. S. (2011). Roles of Brand Value Perception in the Development of Brand Credibility and Brand Prestige. 2011 ICHRIE Conference, 13, 1-8. www.googlescholar.com

Pecot, F., \& De Barnier, V. (2018). Brands using historical references: a consumers' perspective. Journal of Brand Management, 25(2), 171-184. https://doi.org/10.1057/s41262-017-0076-y

Phillip, K., Gus, M., Rodney, A., \& John, A. (2003). Customer repurchase intention. European Journal of Marketing, 37(11), 1762-1800.

Prasad, K., \& Dev, C. S. (2000). Managing Hotel Brand Equity. Cornell Hotel and Restaurant 
Administration Quarterly, 4l(3), 22-31. https://doi.org/10.1177/001088040004100314

Ramírez, P. E., Mariano, A. M., \& Salazar, E. A. (2014). Propuesta Metodológica para aplicar modelos de ecuaciones estructurales con PLS: El caso del uso de bases de datos científicas en estudiantes universitarios. Revista ADMpg Gestão Estratégica, 7(2), 133-139. http://www.admpg.com.br/revista2014_2/Artigos/15\%20\%20-\%20Artigo_15.pdf

Roberts, J. H., \& Lattin, J. M. (1991). Development and Testing of a Model of Consideration Set Composition. Journal of Marketing Research, 28(4), 429. https://doi.org/10.2307/3172783

Sataloff, R. T., Johns, M. M., \& Kost, K. M. (2001). Evaluating Relationships among Brand Experience, Brand Personality, Brand Prestige, Brand Relationship Quality, and Brand Loyalty: An Empirical Study of Coffeehouse Brands. Hospitality Management and Dietetics Kansas State University, 2(2001). https://scholarworks.umass.edu/cgi/viewcontent.cgi?article=1283\&context=gradconf_hospit ality

Sirieix, K.; Dubois, P. L. (1999). Vers un modèle qualité-satisfaction intégrant la confiance? Recherches et Applications en Marketing, 14.

Taasoobshirazi, G., \& Wang, S. (2016). The performance of the SRMR, RMSEA, CFI, AND TLI. Journal of Applied Quantative Methods, 11(3), 31-41.

Torres Valverde, E. ; P. R. G. (2013). Medición de la intención de compra con base en un modelo de regresión logística de productos de consumo masivo. Universidad politécnica Salesiana sede Quito.

Wang, H. J. (2017). Determinants of consumers' purchase behaviour towards green brands. Service Industries Journal, 37(13-14), 896-918. https://doi.org/10.1080/02642069.2017.1365140

Wang, J., Tao, J., \& Chu, M. (2020). Behind the label: Chinese consumers' trust in food certification and the effect of perceived quality on purchase intention. Food Control, 108(August 2019). https://doi.org/10.1016/j.foodcont.2019.106825

Wu, K., Chen, S., \& Yuan, Y. (2018). Research on the customer loyalty of bicycle-sharing company based on PLS-SEM model. ACM International Conference Proceeding Series, 6872. https://doi.org/10.1145/3180374.3181358

Xu, P. (2019). College Students' Perceived Brand Value in Service and Manufacturing Categories. Iciss. https://doi.org/10.25236/iciss.2019.024 